\title{
Algunas consideraciones sobre la estructura curricular de los programas de Economía en Colombia ${ }^{1}$
}

\section{Some Considerations about the Structure of Economics Curriculums in Colombia}

\section{Algumas considerações sobre a estrutura curricular dos programas de Economia em Colômbia}

\author{
Javier Andrés Castro Heredia ${ }^{2}$ \\ Docente e investigador de la Universidad del Valle, Cali, Colombia \\ javier.castro@correounivalle.edu.co
}

\section{Leonardo Raffo López ${ }^{3}$}

Docente e investigador de la Universidad del Valle, Cali, Colombia

leonardo.raffo@correounivalle.edu.co

Recibido: $29 / 06 / 2015$

Aceptado: $24 / 11 / 2015$

1 Resultado del proyecto de investigación Estado de la Enseñanza de la Economía en Colombia financiado por la Vicerrectoría de Investigaciones de la Universidad del Valle.

2 Economista. Magíster en Economía.

3 Economista. Magíster en Economía aplicada. 


\title{
Resumen
}

Este artículo expone algunas particularidades de las estructuras curriculares de los programas de Economía a partir del análisis cuantitativo de una muestra seleccionada con criterios de región y rendimiento en los exámenes de estado para los estudiantes de Economía. De acuerdo con los resultados de los análisis de la estructura curricular de los programas de Economía, se observan convergencias entre las estructuras curriculares con unas pequeñas diferencias regionales. Esta creciente homogenización de currículos puede sugerir la existencia de una estrategia de líder-seguidor dentro de los programas de Economía. Los programas tienden a imitar la estandarización de unos programas líderes nacionales para ofrecer currículos modernos, competentes, prestigiosos o de renombre, flexibles e integrales sin tener en cuenta sus propias especificidades o sellos diferenciadores.

Palabras clave: enseñanza de economía; pregrado; estructuras curriculares; educación superior; Colombia.

\section{Clasificación JEL: A220.}

\begin{abstract}
This paper presents some peculiarities about economics undergraduate programs in Colombia with a quantitative analysis of a selected sample according to two aspects, region and student's performance in a test of economics knowledge. The analysis shows convergence in curricular structures of Economics programs, with small regional differences. This standardization of curricula may suggest the existence of a leader-follower strategy in economics programs. Some programs tend to imitate standardization of national leading programs to provide modern, prestigious, flexible and comprehensive curricula regardless their own particular specificities and features.
\end{abstract}

Keywords: teaching economics; undergraduate; curricula; higher education; Colombia.

\section{Resumo}

Este artigo apresenta algumas especificidades curriculares dos programas de ensino de economia, a partir de uma amostra selecionada com critérios de região e desempenho acadêmico dos estudantes de Economia nos exames estatais. A análise evidencia uma convergência nas estruturas curriculares, com pequenas diferenças regionais. Essa padronização dos currículos sugere a existência de uma estratégia líder-seguidor nos cursos de graduação de economia, tendente a estandardizar-se. Os programas tendem a imitar a estandardização com o intuito de oferecer programas modernos, competentes, prestigiosos, flexíveis e abrangentes, sem ter em vista suas particularidades.

Palavras-chave: ensino de economia; graduação; estruturas curriculares; educação superior; Colômbia.

\footnotetext{
¿Cómo citar este artículo? / How to quote this article?

Castro, Javier y Leonardo Raffo. «Algunas consideraciones sobre la estructura curricular de los programas de Economía en Colombia». Sociedad y economía, No. 30 (enero - junio de 2016): 147-170.
} 


\section{Introducción}

El objetivo de este trabajo es exponer algunas particularidades de las estructuras curriculares de los programas de Economía en Colombia, a partir de una muestra seleccionada con criterios de región y rendimiento en los exámenes de estado (ECAES) para los estudiantes de economía. Las particularidades analizadas corresponden a la participación de las áreas temáticas en el currículo de los programas de Economía teniendo en cuenta el tipo de institución de educación superior que brinda el programa, la localización geográfica y su condición de acreditación de alta calidad.

La creciente homogeneización de las estructuras curriculares de los programas de Economía en Colombia, detectada desde hace más de tres lustros por González (1999) y confirmada más recientemente por Lora y Ñopo (2009), es una de las motivaciones del presente trabajo. Aquí se contribuye a la discusión sobre la enseñanza de la economía en Colombia mostrando que existe convergencia en la duración de los programas y el número total de asignaturas de los programas de Economía 4 .

Los hallazgos son consistentes con los de Lora y Ñopo (2009) para algunos países de América Latina. Además, existen patrones similares en la conformación y estructura de las áreas de microeconomía, macroeconomía y cuantitativa para los programas de Economía en Colombia. Sin embargo, están presentes algunas diferencias regionales y en las estructuras de las áreas de pensamiento e historia económica que sugieren la existencia de algunas especificidades y rasgos propios en los programas analizados.

Este artículo apunta a la reflexión acerca del papel que han desempeñado los procesos de acreditación de alta calidad del Consejo Nacional de Acreditación (CNA) y la influencia de las universidades de mayor prestigio y tradición en los procesos de convergencia de los currículos de economía en Colombia. Aparte de la presente introducción el artículo se ha organizado de la siguiente manera: en la primera sección se exponen algunos antecedentes en la materia para Latinoamérica y Colombia; en la segunda se expone el contexto de la investigación; en la tercera se precisa la metodología del estudio; en la cuarta se exponen los principales resultados obtenidos. Por último, se plantean algunos comentarios finales a manera de conclusión.

\section{Algunos antecedentes}

\subsection{La enseñanza de la economía en Latinoamérica}

La enseñanza de la economía es un tema que suscita bastante polémica debido a las diversas posiciones y escuelas existentes, pero no es tan frecuente su interés en él por parte de la mayoría de los economistas académicos. No obstante, en el caso latinoamericano durante los últimos años se ha suscitado una

4 Se podría utilizar el número de créditos por asignatura y totales del programa que tienen una correlación por encima de 0,90 para la muestra seleccionada. Sin embargo, por falta de disponibilidad de alguna información correspondiente a unos cuantos programas de la muestra y la necesaria comparabilidad con trabajos anteriores, se maneja el número de asignaturas de los programas. 
renovada disposición por establecer y sostener el debate académico en torno a este tema. Los trabajos del BID en esta materia han avanzado notablemente en este sentido. Es así como Lora y Ñopo (2009) muestran que el estado de la enseñanza en algunos países de América Latina es relativamente estándar, pero carece de un mayor carácter profesionalizante, de la formación en competencias básicas y conocimientos sobre la sociedad y la política contemporáneas, todo lo cual es útil para el desempeño laboral. Es decir, a los mencionados autores les interesa mostrar la disociación que existe entre lo que se enseña en economía y lo que necesitan los egresados de economía en el mundo laboral.

Siguiendo esa misma línea de investigación, se han realizado trabajos específicos para varios países latinoamericanos: Argentina, Bolivia, Chile, México, Uruguay y Colombia. Para el caso argentino, Rozenwurcel, Bezchinsky y Rodríguez (2009) examinan cuatro grandes áreas temáticas en los programas de Economía y afirman que no existe consenso general acerca del predominio del enfoque neoclásico en la formación del economista argentino. Esto se puede deber al enorme peso (40\%) de la Universidad de Buenos Aires (UBA) en el total de estudiantes matriculados en ese país, y a la alta heterogeneidad en los procesos de formación y enseñanza ofrecidos en esa universidad.

Como señala Hodara (2010), a pesar de que la estructura curricular básica no es muy disímil entre las universidades públicas y las privadas, algunas diferencias se han heredado del pasado. Este autor menciona que mientras en la UBA son obligatorias las asignaturas de sociología, metodología de las ciencias sociales y epistemología de la economía - las cuales no se dictan en otras universidades argentinas-, en el programa de Economía de la Universidad Torcuato di Tella (UTDT) únicamente se ofrece un curso obligatorio de problemas filosóficos y uno de escritura.

Para Bolivia, Espinoza, Machicado y Makhlouf (2009) encuentran que existe una brecha entre la enseñanza que ofrecen las universidades privadas y las públicas, en especial entre la Universidad Mayor de San Andrés -la cual es pública- y el resto de universidades. Uno de los problemas estructurales identificados en el estudio es que la malla curricular de los programas de Economía de universidades públicas - según los autores- es obsoleta, al tiempo que no hay homogeneidad en las cátedras para un mismo semestre.

Generalmente, en Bolivia los profesores de economía no son de tiempo completo, y en las universidades públicas no se estimula el trabajo investigativo de los estudiantes; de hecho, el mayor problema de los departamentos de Economía es el bajo desarrollo de la actividad investigativa.

Por otra parte, las perspectivas económicas de los egresados -según el estudio referido- tampoco son buenas. La mayor parte de los economistas sin título de posgrado en ese país son mal pagos (Espinoza, Machicado y Makhlouf 2009):

\footnotetext{
Existen también serios problemas de incentivos en Bolivia, donde además de que los economistas son muy mal pagados, son peor pagados aquellos que se dedican a la docencia, el $66,7 \%$ de los encuestados ganan menos de US $\$ 500$ como profesores. En cambio en Chile un $\mathrm{PhD}$ que por lo general trabaja como profesor a tiempo completo no gana menos de US\$2,500. Evidentemente las condiciones económicas así como el nivel de vida de cada país, son diferentes, pero aún así no es una buena señal que el $50 \%$ de los economistas con maestría en Bolivia, ganen en promedio entre US\$500 y US\$1,000 al mes $(2009,85)$.
} 
El panorama descrito para Bolivia contrasta con el que se observa para Chile, donde la estructura curricular tiende a ser homogénea, en especial en los cursos básicos. No obstante, se observan diferencias en los cursos electivos. La ventaja relativa de los programas de Economía en Chile estriba en que la mayor parte de sus profesores son de tiempo completo y poseen título de doctor (Espinoza, Machicado y Makhlouf 2009); esto, sin duda, influye positivamente en la investigación, en el número de publicaciones de los profesores y en la formación investigativa de los estudiantes, tanto de pregrado como de posgrado. Todo lo anterior redunda en que los bolivianos perciben los programas de Economía chilenos como un modelo de enseñanza a seguir, lo que en la práctica se traduce en que un gran número de bolivianos adelantan estudios de posgrados en el país vecino (Espinoza, Machicado y Makhlouf 2009).

El trabajo de Ahumada y Butler (2009) encuentra que en México ha disminuido la matrícula de estudiantes de Licenciatura en Economía de las universidades seleccionadas, y que se privilegia la enseñanza estándar del enfoque neoclásico. No obstante, -según los autores- falta trabajar mucho más en la inserción de los estudiantes al manejo de los programas de computador y los recursos de internet en la enseñanza.

Para el caso de Uruguay, Hodara (2010) señala que la Universidad de la República concentra la mayor parte de los estudiantes que ingresaron $(72 \%)^{5}$, matriculados (85\%) y egresados (58\%) de Licenciatura en Economía en ese país. Sin embargo, -según este autor- se aprecia una menor eficiencia en la formación de los economistas por parte de esta universidad. Aunque Hodara no argumenta explícitamente su afirmación, es evidente que se apoya en que la proporción de ingresos sobre egresos del programa de Licenciatura en Economía es relativamente alta, alrededor de $35 \%$, según sus datos.

Cabe resaltar que en contravía con la tendencia convergente en los currículos de economía hallada en nuestro trabajo y del de Lora y Ñopo (2009), Hodara (2010) advierte que la diversidad entre los distintos países de América Latina es mayor que entre el promedio de estos y los países desarrollados: "Este hecho pone una cota a los análisis basados exclusiva o principalmente en generalización regionales" $(2010,17)$.

\subsection{La enseñanza de la economía en Colombia}

Para el caso colombiano se han desarrollado pocos trabajos, pero todos ellos señalan la importancia de las características y prácticas de los programas en la formación de los economistas. Bejarano (1984), Kalmanovitz (1999) y Salazar (2010) coinciden en la necesidad de perfeccionar la enseñanza de la economía en el país. Es cierto que ha habido transformaciones en los currículos y en la enseñanza desde que Currie (1965) sugirió la necesidad apremiante de consolidar una preparación básica en teoría económica "en los términos más sencillos y menos técnicos que fuera posible" (Currie 1993, 352), con aplicación a problemas reales,

5 Cabe observar que en su trabajo, Hodara (2010) aclara que el número de ingresos a Economía se estimó "suponiendo que en los ingresados y matriculados se mantiene la misma proporción entre Economía, Contador [sic] y Licenciatura en Administración” (2010, 14). 
confundiendo, estimulando y provocando a los estudiantes hasta que logren pensar en su proceso de aprendizaje (Currie 1993)6. Sin embargo, para Bejarano (1984) los economistas no quieren hablar del método que usan, que desconoce realidades complejas y se deja de lado la erudición y el conocimiento amplio para solo formar economistas especialistas y disciplinares.

Es más, para Salazar (2010) existe un núcleo dominante en la enseñanza y la disciplina económica que es consistente y fuerte pero que niega la realidad. Para eso propone un aprendizaje cambiante y complejo, un escenario donde la interacción estudiante-profesor sea mayor y la creatividad para plantearse hipótesis y cuestionamientos sea estimulante. También coincide con Bejarano en la necesidad de ampliar el bagaje cultural, político y social de los estudiantes con la lectura de literatura contemporánea y la escritura. En el mismo sentido, Kalmanovitz (1999) propone métodos alternativos de evaluación, más allá de recitar modelos y recetas, y que los programas sean más intensos en escritura y lectura.

Por esa misma vía, González (1999) -inspirado en Sábato (1979)- advierte que más allá de los currículos y de los programas de los cursos, en el centro de la formación se encuentra el buen docente como maestro, pues es quien "logra despertar el asombro en el estudiante" (54). Con respecto al proceso de enseñanza, González (1999) afirma, en línea con Bejarano (1984), que el desafío radica en mejorar la erudición de los estudiantes sin incluir más asignaturas; pero, en oposición al mismo, aduce que la búsqueda de la erudición no solo debe girar en torno a temas y problemas económicos, en la medida en que algunos temas deberían tratarse con cierta autonomía de los asuntos que preocupan a la disciplina. Por lo tanto, no es suficiente con fomentar una transdiciplinariedad en donde la economía actúa como la "disciplina diagonal".

Para lograr lo anterior, se considera contar con profesores que no sólo sean expertos en economía y en sus campos específicos de trabajo, sino que también sean verdaderos eruditos, con habilidades intelectuales y bagaje suficientes para superar los límites de la disciplina y, de esa manera, ser capaces de motivar en sus estudiantes la pasión por la investigación científica (en ciencias naturales y sociales), las humanidades y las artes.

De esta forma durante los primeros semestres de la carrera, aparte de los cursos básicos de economía, deberían abrirse caminos hacia la multidisciplinariedad y la interdisciplinariedad. A ese respecto, D'Autume (2000) sugiere abrirles un lugar más amplio a la Sociología, a la Historia o a las Ciencias Políticas durante los primeros años de la carrera. La creación de ciclos comunes para ciencias sociales durante los primeros semestres de la carrera puede ser útil en ese sentido. Esto está a tono con las tendencias internacionales recientes de disciplina: recientemente Fourcade, Ollion y Algan (2015) han planteado que aunque la economía ha sido tradicionalmente una disciplina relativamente independiente y con comunidades académicas bastante cohesionadas y jerárquicas, en la actualidad se está abriendo a otras disciplinas como la sociología, las finanzas, la física y las ciencias políticas.

6 En ese orden de ideas, Currie $(1965 ; 1993)$ resalta la importancia de aplicar de forma habilidosa y paciente el método socrático de enseñanza. 


\subsection{Los currículos de economía en Colombia}

A finales del siglo XX ya eran evidentes ciertos síntomas de homogeneización en los currículos de economía en Colombia. En su estudio, González (1999) encuentra que las asignaturas vertebrales del núcleo básico de los programas de Economía en Colombia son: principios, fundamentos o introducción a la economía, microeconomía, macroeconomía e introducción a la economía, con un número de cursos de micro y macroeconomía que oscila entre 3 y 4, y un número de cursos de principios que rara vez llega a dos (González 1999). En sus propias palabras: "Es la secuencia que se desprende de los postulados de la corriente principal y que ha sido asimilada gustosamente por las facultades más prestigiosas del mundo" (1999, 70).

Una corriente principal que en el campo de la microeconomía se viene expresando desde hace más de medio siglo a través del programa de investigación neowalrasiano (González 1999; Raffo y Castro 2011), y más recientemente, en términos paradigmáticos a través de todo el cuerpo teórico que ha sido denominado "neoclasicismo extendido" (Cataño 2004; D’Autume 2000).

Mientras tanto, en el campo de la macroeconomía la corriente principal se ha centrado desde hace por lo menos dos décadas en la "nueva macroeconomía clásica" (González 1999) y también la "nueva macroeconomía keynesiana", desarrollos que heurísticamente llevaron a la consolidación de la teoría macroeconómica de equilibrio general dinámico estocástico (Raffo y Castro 2011).

Con la llegada del siglo XXI, en Colombia se vio la necesidad de consolidar un sistema de aseguramiento de la calidad de la educación con el propósito de ponerse a tono con las transformaciones hacia la estandarización y la globalización de la educación superior que se venían dando internacionalmente desde los años de 1990; es así como fue creada la Comisión Nacional Intersectorial de Aseguramiento de la Calidad de la Educación (Conaces) mediante el Decreto 2230 de 2003.

Esto cambió las reglas del juego de los programas de Economía y de todos los programas de educación superior en Colombia. La puesta en práctica de los exámenes ECAES para los egresados, así como de los procesos de registro calificado y acreditación de los programas, como resultado de lo anterior, configuró un nuevo sistema de acreditación y señalización de la calidad, que ha jalonado una tendencia mucho más fuerte hacia la estandarización tanto de los currículos como de los programas de los cursos, los manuales, algunas posiciones ideológicas y hasta del tipo de docentes (Cuenca 2010). Esto es resultado de la consolidación internacional de una serie de tendencias educativas y pedagógicas dominantes.

En el campo pedagógico, el análisis de competencias y estándares se ha convertido en un proceso central en la educación en diferentes regiones y países, al igual que en los proyectos y acciones promovidos por organismos internacionales (De Camilloni 2009). Como aduce esta autora, desde sus orígenes el empleo de la estandarización ha estado ligado a la medición cuantitativa, lo que -según advierte agudamente- "tiene como grave consecuencia el estrechamiento de los propósitos de la educación" (2009, 67). 
Resultado de su análisis, Cuenca (2010) concluye que la enseñanza debe partir de la enseñanza de la teoría del equilibrio general; de hecho sugiere que si esta teoría es el núcleo de la corriente principal - lo que efectivamente ha sido planteado por Weintraub (1985) y otros al analizar la estructura del programa de investigación neowalrasiano desde una perspectiva lakatosiana-, entonces debería enseñarse como un curso independiente. Además, para la autora debe fortalecerse la formación teórica de los estudiantes a través de cursos de pensamiento económico. Por último, ella añade que debe proponerse "la necesidad de incluir también la historia, la sociología e incluso la psicología en los estudios posteriores de la economía" (2010, 146).

En un estudio más reciente, Sarmiento y Silva (2014) estudian las características de la educación recibida por los estudiantes de economía en Colombia. Con ese propósito realizan un análisis de las condiciones socio-económicas, las preferencias y el desenvolvimiento en la carrera, al igual que el marco institucional de una muestra de estudiantes de economía durante el 2007. Entre otros hallazgos, estos autores encontraron que hay homogeneidad en las instituciones en cuanto al número de cursos ofrecidos para la mayor parte de las áreas básicas a excepción de macroeconomía, historia económica y pensamiento económico:

\footnotetext{
Se encontró que hay cierta homogeneidad en las instituciones en cuanto al número de asignaturas de Introducción a la Economía (1, 73,58\%), Microeconomía (24, 90,57\%), Estadística (2, 77,36\%), Econometría (2, 67,92\%) y Cuantitativas (4-5, $67,92 \%)$. Del mismo modo, en cuanto a otras asignaturas complementarias a la formación integral del economista, en el 2007 los planes de estudio no contenían asignaturas como Idiomas $(56,60 \%)$ y Sistemas $(66,04 \%)$.
}

Sin embargo, no hay un consenso claro en el número de asignaturas como Macroeconomía (7-16), Historia Económica (0-3), Pensamiento Económico (0-2), Electivas de profundización (2-9), Contabilidad y Finanzas (2-8), Socio-humanísticas (3-10), Administrativas (0-3), Derecho (0-2), Investigación (0-4), Prácticas y/o Trabajos de Grado de carácter obligatorio (0-2) $(2014,239)$.

Por otra parte, Sarmiento y Silva (2014) encuentran que en el 2007, de un total de 49 instituciones el mayor porcentaje de programas con carga completa tiene una duración de 10 semestres (para 57,14\% de los programas), a la que le sigue una de 9 semestres (para 34,69\% de estos) y una de 8 semestres (para 8,16\%); para los programas acreditados encuentran una duración promedio de la carrera relativamente menor que la de los programas no acreditados. Esto último como se verá es consistente con nuestros hallazgos. Por último, cabe agregar que estos autores identificaron que las instituciones con acreditación exhiben un número promedio de monitores por área mayor que las instituciones no acreditadas.

\section{El contexto: los programas de Economía en Colombia (2000-2012)}

La base de datos del Sistema Nacional de Información para la Educación Superior (SNIES) del Ministerio de Educación Nacional (MEN) muestra la existencia de 101 programas de Economía en toda Colombia para el segundo semestre 
de 2012. Estos registros incluyen los programas a distancia o virtuales y las extensiones de los programas a otras sedes de las universidades.

Para ese período de tiempo se observan 77 programas de Economía activos, es decir, con estudiantes matriculados y con registro calificado, de los cuales 27 corresponden a universidades públicas y 50 a universidades privadas. Esos programas en su mayoría son de carácter presencial (90\%) y se encuentran situados en las tres principales ciudades del país (52\%). En Bogotá se encuentran 25 programas de Economía (33\%), de los cuales tres pertenecen a instituciones públicas.

Durante el período de 2010 a 2012 se crearon en Colombia 14 nuevos programas de Economía: 28,5\% corresponde a universidades públicas, que inauguraron programas en Neiva (Universidad Surcolombiana en 2005-2), Bogotá (Colegio Mayor de Cundinamarca en 2006-2), Sincelejo (Universidad de Sucre en 2008-2) y Armenia (Universidad del Quindío en 2010-2). Las universidades privadas crearon diez nuevos programas de Economía principalmente en la Costa Atlántica (4) y en Bogotá (3) ${ }^{7}$. En general, en Bogotá es donde más se han creado programas de Economía en el período 2000-2012, con un total de cuatro programas. Sigue la región de la Costa Atlántica, también con cuatro programas, no en las ciudades tradicionales sino, dos en Sincelejo, uno en Montería y uno en Montelíbano (Córdoba).

En la base de datos del SNIES se encuentran 24 programas de Economía que se pueden catalogar de inactivos, dado que no presentan matriculados ni entrada de nuevas cohortes de estudiantes para el segundo semestre de 2012. También se observa que 13 programas inactivos pertenecen a instituciones privadas y 11 a universidades públicas. Estos últimos corresponden a extensiones del programa de Economía a sedes regionales, por ejemplo, en el caso de la Universidad de Nariño en Ipiales, el de la Universidad de los Llanos en San José del Guaviare, o en el de los programas a distancia de la Universidad de Pamplona en Norte de Santander. En el caso de las universidades privadas, se pueden observar algunas desapariciones de sucursales de programas o descontinuación y cambios de nombres, como es el caso de la EAN en Bogotá y la Universidad Pontificia Bolivariana en Medellín.

Los datos de estudiantes matriculados del Ministerio de Educación Nacional, para el período 2000-1 a 2012-2, muestran tres fases en la evolución de la magnitud de los estudiantes de economía, tal como se puede apreciar en la gráfica 1. En primer lugar, se observa una fase de decrecimiento en la matrícula total de estudiantes a una tasa promedio anual de $-1,9 \%$ entre 2000 y 2004 . Luego, se nota una fase de recuperación de la matrícula entre 2005 y 2009 del 1,7\% promedio anual, llegando hasta 18.218 estudiantes de economía matriculados en los programas oficiales y privados. En tercer lugar, se advierte una fase de estabilización para los dos últimos años (2010-2012) del período analizado, con una tasa de crecimiento promedio anual de la matrícula apenas positiva $(0,4 \%)$.

7 Los programas de Economía creados por universidades privadas desde el 2000 son: CECAR de Sincelejo (2000-2), Universidad del Norte (2001-1), Unitrópico de Yopal (2003-1), Universidad Autónoma de Bucaramanga (2005-1), EAN de Bogotá (2006-1), ICESI de Cali (Economía y Políticas Públicas, 2006-2), Universidad Pontificia Bolivariana UPB de Montería (2007-1), Universidad de la Sabana de Bogotá (2008-2), Politécnico de Bogotá (2009-1) y Universidad Pontificia Bolivariana UPB de Montelíbano (2011-1). 
Gráfica 1. Evolución del número de matriculados en los programas de Economía 2000-2012

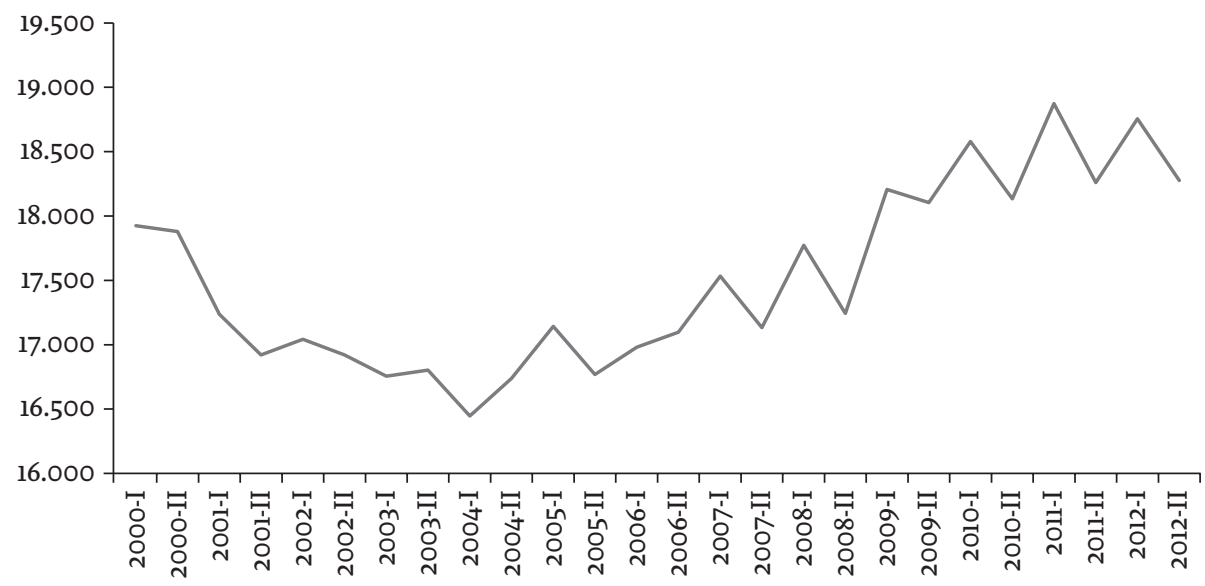

Fuente: elaboración propia con base en SNIES, Ministerio de Educación Nacional (2014).

En su análisis de largo plazo entre 1946 y 2007, Sarmiento y Silva (2014) muestran que el número de graduados tuvo un crecimiento significativo a partir de los años de 1970; se detecta que el número de graduados ha exhibido dos tendencias decrecientes en dos períodos: 1988-1993 y 2002-2007 (Sarmiento y Silva 2014). Claramente, el último período de descenso en los graduados es una consecuencia de la baja detectada en el número de matriculados entre el 2000 y el 2004 .

Sin embargo, existen diferentes trayectorias en el número de matriculados en programas de Economía de acuerdo con el tipo de institución (oficial o privada). Por un lado, la matrícula de los programas de Economía de las universidades oficiales creció durante todo el período analizado (2000-2012). Tuvo una fase de aceleración durante los cinco primeros años y luego sostuvo los niveles de crecimiento en la matrícula alrededor de 1,5\% promedio anual. Por otro lado, la matrícula en las universidades privadas presentó una tendencia decreciente en los cinco primeros años para luego estabilizarse y contar con episodios de crecimiento moderado de $2 \%$ (2005-2009) y decrecimiento de -0,5\% (2010-2012). Estas cifras muestran la reducción sistemática de la brecha de matrícula entre los programas de instituciones privadas y oficiales, y la presencia de un proceso de convergencia hacia un nivel aparentemente estable en el número total de estudiantes de economía a escala nacional, tal como se presenta en la gráfica 2.

Otra peculiaridad de los datos agregados de matrícula se relaciona con el comportamiento de la matrícula de estudiantes de economía con respecto al total de matrícula de estudiantes en carreras profesionales en Colombia para el período 2000-2012. Tal como se aprecia en la gráfica 3, la participación de los matriculados en Economía cada vez es menor, pasando de 2,5\% en el 2000 a 1,5\% durante el 2012. Este descenso en la participación relativa es sostenido, dado que el crecimiento promedio anual de la matrícula total en programas profesionales es $4,3 \%$ frente a un pequeño $0,3 \%$ de la matrícula de economía durante el período 2000-2012. 
Gráfica 2. Evolución del número de matriculados en los programas de Economía por

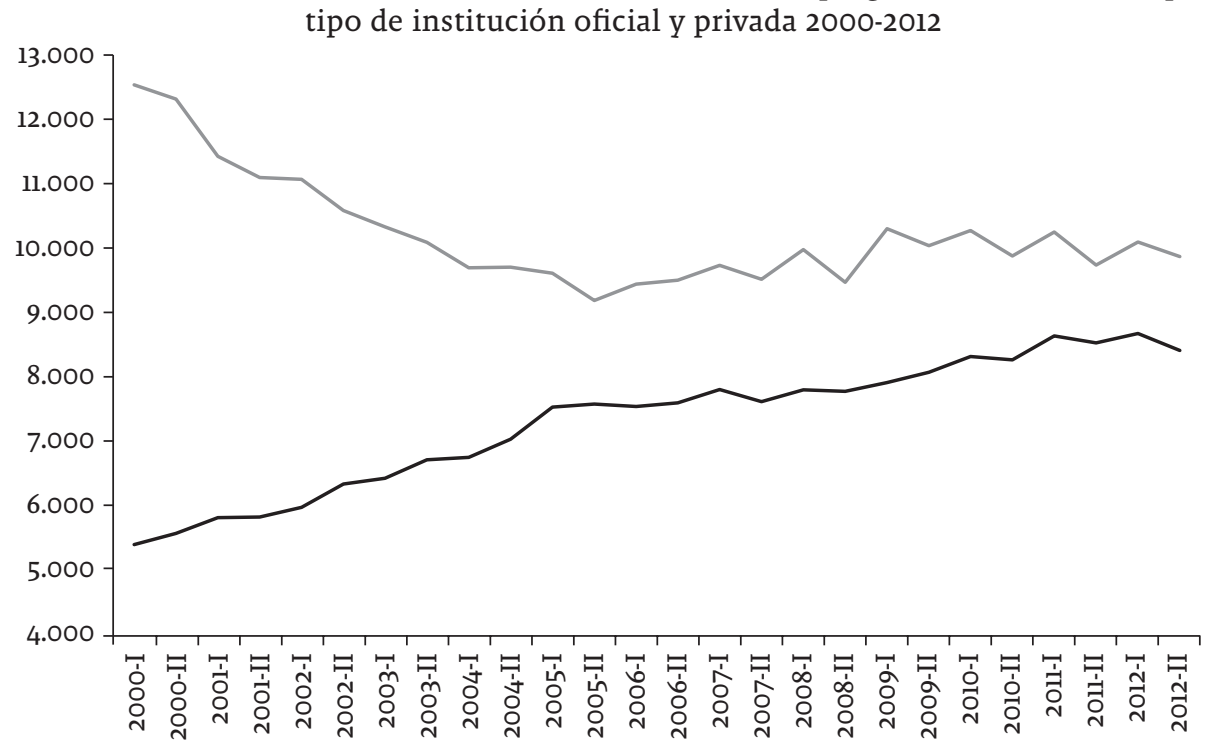

- Oficial Privado

Fuente: elaboración propia con base en SNIES, Ministerio de Educación Nacional (2014).

Gráfica 3. Evolución de la participación de los estudiantes matriculados en los programas de Economía en el total de matriculados en programas profesionales en Colombia

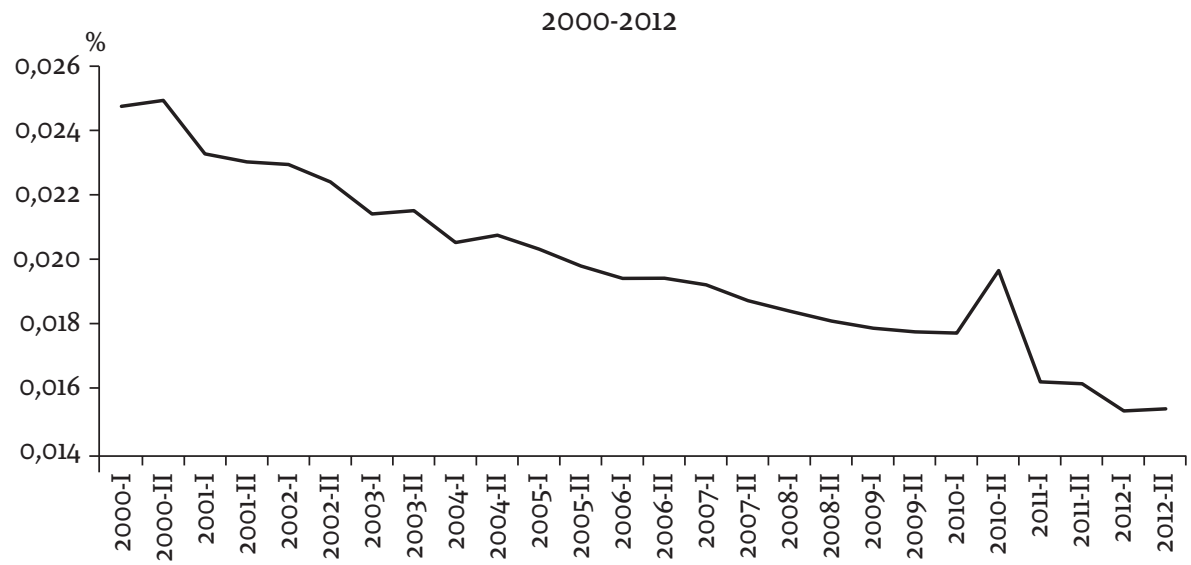

Fuente: elaboración propia con base en SNIES, Ministerio de Educación Nacional (2014).

Por lo anterior, la baja observada en el número de matriculados en economía entre el 2000 y el 2004 probablemente se explica por un cambio en las preferencias de los jóvenes hacia los distintos programas académicos ofrecidos en Colombia y la diversificación de otras carreras afines a la economía (Sarmiento y Silva 2014). Estos cambios llevaron a que los aspirantes a ingresar a la educación superior tuvieran menor preferencia por los programas de Economía en 
comparación con otros programas afines como Administración de Empresas, Contaduría Pública, Comercio Exterior, Negocios Internacionales, Finanzas; y también con otros menos afines como Ingeniería Industrial, Estadística-Matemática, Ciencias Políticas-Relaciones Internacionales, entre otros, todos ellos de corte más técnico y, posiblemente, menos complejos y con mejores perspectivas laborales futuras desde la perspectiva de los jóvenes.

La recesión económica que atravesó la economía colombiana en 1998 pudo haber sido un factor detonante en los cambios en las preferencias de los jóvenes. Pero, aparte de esta, las tendencias globales de la economía mundial tendientes a crear una mayor demanda de ocupaciones técnicas ligadas a los procesos de tercerización y flexibilización laboral, donde son esenciales los procesos de outsourcing y offshoring comandados por corporaciones multinacionales, pueden haber creado estímulos para ingresar a programas sustitutos de naturaleza más técnica y aplicada que la economía, como los mencionados arriba.

A esto se suma la ampliación de la oferta educativa, del número de matriculados y la aparición de nuevos programas académicos afines a partir del año 2000 (Sarmiento y Silva 2014), tendencia que también ha sido constatada en toda América Latina durante las dos últimas décadas (Villanueva 2008). Al respecto, Sarmiento y Silva (2014) muestran que para el período que va del primer semestre de 2000 al primer semestre de 2006 el número de matriculados disminuyó para Economía en 12\%, mientras que también descendió para las carreras afines como Administración y Contaduría aunque solo en 4,81\% y 7,52\% respectivamente; pero al mismo tiempo aumentó notablemente para otras carreras menos afines a la Economía como Ciencia Política y Relaciones Internacionales (165,02\%), Ingeniería Industrial (9,37\%) y Matemáticas-Estadística (29,61\%).

Las siguientes gráficas ilustran la evolución de la participación de los estudiantes matriculados para las carreras de Administración de Empresas (gráfica 4) y Contaduría Pública (gráfica 5) en Colombia.

Gráfica 4. Evolución de la participación de los estudiantes matriculados en los programas de Administración de Empresas en el total de matriculados en programas

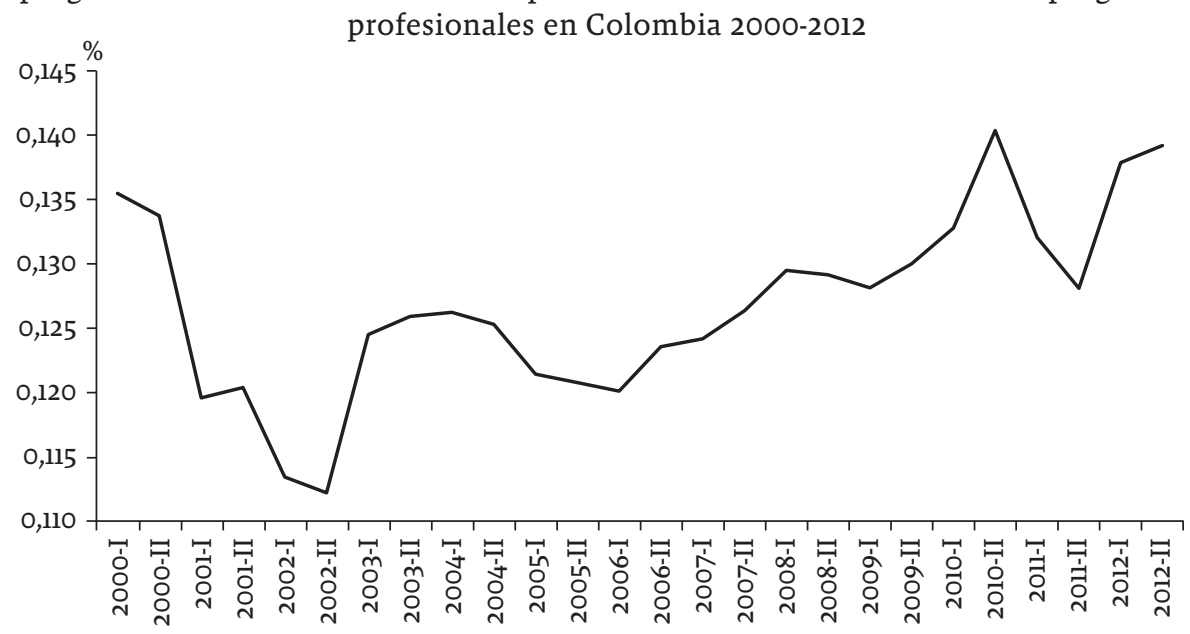

Fuente: SNIES, Ministerio de Educación Nacional. 
Gráfica 5. Evolución de la participación de los estudiantes matriculados en los programas de Contaduría Pública en el total de matriculados en programas

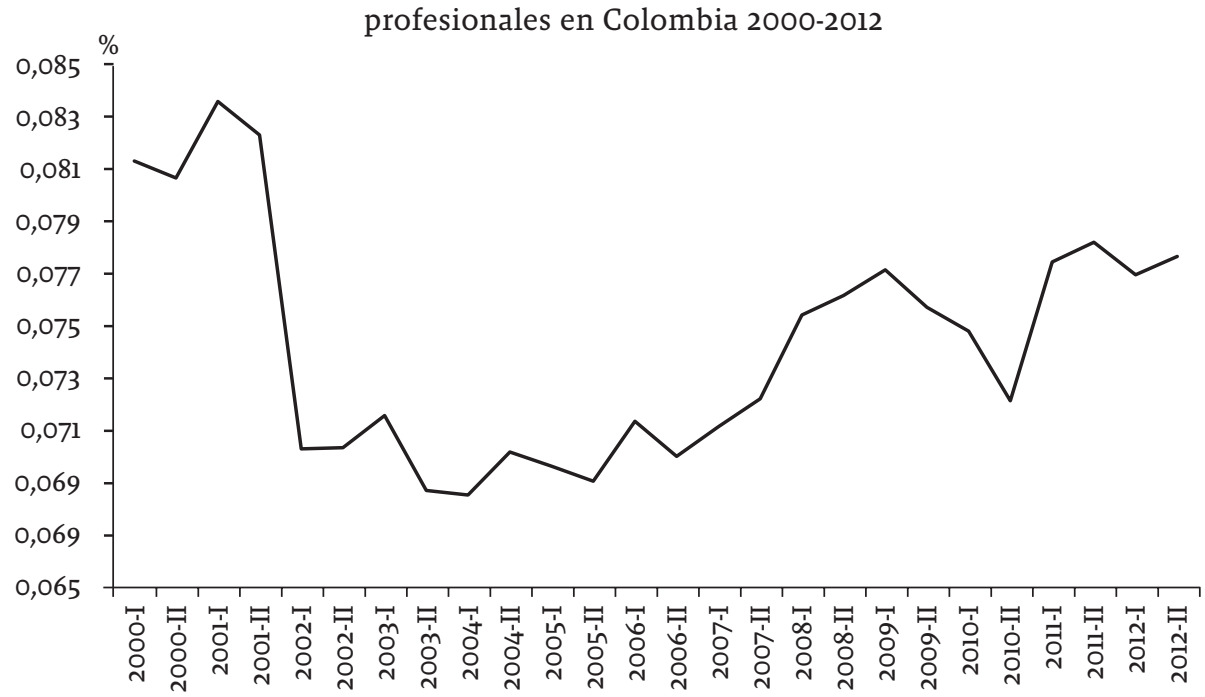

Fuente: SNIES, Ministerio de Educación Nacional.

Estas gráficas hacen constar que estas dos carreras afines también exhibieron tendencias claramente decrecientes en la participación del número de estudiantes matriculados en ellas frente al total de matriculados, pero solo entre el primer semestre del 2000 y el segundo semestre del 2002, probablemente por el impacto de la recesión que atravesó la economía colombiana al final de la última década del siglo XX.

Al mismo tiempo muestran cómo a partir del 2003 la misma variable comenzó a tener una tendencia marcadamente creciente para ambos programas. Esto corrobora el cambio en las preferencias de los estudiantes hacia estas carreras, especialmente a partir del 2003, a la par de una mayor preferencia hacia otros programas, algunos afines, otros no tan cercanos como aducen Sarmiento y Silva (2014).

\section{Metodología}

En este trabajo se diseñó una propuesta metodológica para establecer una muestra de los programas de Economía a analizar. Es necesario tener criterios formales para la escogencia de la muestra, dado que eso asegura representatividad académica y regional para fortalecer los hallazgos y el contraste de hipótesis de trabajo.

En primer lugar, se definieron unas regiones geográficas para referenciar y ubicar espacialmente los programas de Economía de todo el país. Se buscó especificar y formar regiones que agrupen departamentos tomando algunas características comunes, como la cercanía y convenciones tradicionales en el manejo de regionalización en Colombia. 
Las regiones que se establecieron fueron: Costa Atlántica, Central, Eje Cafetero y Suroccidental. La región Costa Atlántica cubre a los tradicionales siete departamentos de esa región; la región Central incluye los departamentos del centro, el nororiente y oriente del país; la región Eje Cafetero corresponde a los departamentos de Antioquia, Caldas, Quindío y Risaralda; y la región Suroccidental a los departamentos de Valle del Cauca, Cauca, Nariño y Huila.

Una vez localizados los programas de Economía en cada región se puede determinar un peso específico o relativo de cada región en el total nacional. Para esta determinación y cuantificación, además del número de programas de Economía para cada región, se toma el número de graduados de cada programa durante el período 2000-2012. Es decir, se logra tener una participación porcentual de cada región en la formación del economista teniendo en cuenta el número de programas y su impacto en forma de la magnitud de egresados.

El segundo criterio fueron los resultados del examen ECAES desde 2004 a 2010. Estas pruebas corresponden al período de tiempo en el que se evaluó sobre conocimientos específicos en economía. Las áreas evaluadas fueron: macroeconomía, microeconomía, econometría, pensamiento e historia económica. Las pruebas eran de carácter voluntario para los estudiantes de economía pero a partir del 2010 se volvieron obligatorias.

Teniendo en cuenta los resultados de las pruebas para cada programa de Economía, se realizaron ordenamientos o clasificaciones en cada una de las regiones establecidas anteriormente. De esta manera, para cada región se tuvo una lista ordenada de mayor a menor y se pudieron escoger los programas de acuerdo con su ubicación. Es decir, fue posible hacer una muestra intrarregional de acuerdo con el peso relativo de la región, tanto de la parte de arriba de la lista programas con buenos resultados como de la parte de abajo de la lista. Esto asegura una representatividad regional de programas con buenos resultados y con regulares resultados en las pruebas de estado durante el período de análisis.

Adicionalmente, se conformó un listado de los nueve primeros programas de Economía de acuerdo con los resultados del promedio ponderado de las pruebas de estado para el período 2004-2010. Este listado permite hacer otra contrastación y análisis de las estructuras curriculares, a partir de aquellos programas que hemos denominado programas top, por sus altos resultados.

La muestra para el análisis de este trabajo quedó conformada por 34 programas de Economía de un total de 77 programas presenciales existentes durante el período de estudio: seis programas de la Costa Atlántica, trece de la Región Central, seis del Eje Cafetero y nueve de la región Suroccidental. Se tienen ocho programas de Economía adscritos a universidades públicas y 26 de universidades privadas. De la muestra, se tienen 21 programas de Economía acreditados de alta calidad por el Consejo Nacional de Acreditación CNA en la fecha de corte de este trabajo (2013-2) .

8 La muestra de programas de Economía corresponde a las universidades: Pontifica Universidad Javeriana (Bogotá y Cali), Universidad Autónoma de Occidente, Universidad Autónoma Latinoamericana, Universidad Católica de Pereira, Universidad Central, Universidad Cooperativa de Colombia (Bogotá), Universidad de Antioquia, Universidad de Cartagena, Universidad de la Salle, Universidad de los Andes, Universidad de Nariño, Universidad del Cauca, Universidad del Magdalena, Universidad del Norte, Universidad del Rosario, Universidad del Sinú, Universidad 
Con los programas de Economía seleccionados para el análisis se pasó a recopilar y sistematizar la información sobre la estructura curricular, las asignaturas, los contenidos temáticos, metodologías de enseñanza y referencias bibliográficas utilizadas9. La información se obtuvo desde la página web de los distintos programas de Economía, igualmente mediante visitas y correos electrónicos a los directores de programa y jefes de departamento de Economía.

\section{Principales resultados: las estructuras curriculares de los programas de Economía en Colombia}

\subsection{Generalidades}

Los programas de Economía de la muestra presentan una duración parecida. La mediana de duración de los programas es 9 semestres y un promedio de 9,12 semestres con una desviación estándar de 0,69 semestre para el total de la muestra. La duración máxima se encuentra en los 10 semestres y la mínima en 8 semestres. Esto coincide con la apreciación de Lora y Ñopo (2009), quienes sugieren la existencia de una tendencia reciente a tener programas más cortos.

La duración promedio de los programas de universidades privadas (9 semestres) es levemente inferior a la de los programas de las universidades públicas (9,5 semestres). Prácticamente no existen diferencias entre la duración promedio de los programas acreditados $(9,04)$ por el CNA y los no acreditados $(9,25)$. Sin embargo, existen pequeñas diferencias en la duración de los programas por regiones. Tal como se puede apreciar en la tabla l, los programas de la región Costa Atlántica tienen una duración significativamente inferior ( 8,6 semestres) que los de las otras regiones del país tomadas para el presente análisis. Además se observa una desviación estándar pequeña $(0,54)$ lo cual sugiere una menor dispersión entre la duración de los distintos programas de esta región.

Tabla 1. Duración promedio de los programas de Economía por región

\begin{tabular}{|l|c|c|}
\multicolumn{1}{c|}{ Región } & Duración promedio & D. E. \\
\hline Costa Atlántica & 9,30 & 0,50 \\
Central & 9,33 & 0,81 \\
Eje Cafetero & 9,07 & 0,75 \\
Suroccidental & 8,60 & 0,54 \\
\hline
\end{tabular}

Fuente: Base de datos del proyecto Estado de la Enseñanza de la Economía en Colombia, cálculos propios.

del Valle, Universidad EAFIT, Universidad EAN, Universidad Externado de Colombia, Universidad Icesi, Universidad Jorge Tadeo Lozano, Universidad Libre (Cali), Universidad los Libertadores, Universidad Nacional de Colombia (Bogotá y Medellín), Universidad Pontificia Bolivariana, Universidad de San Buenaventura (Cali), Universidad Santiago de Cali, Universidad Santo Tomás (Bogotá), Universidad Sergio Arboleda, Universidad Simón Bolívar y Universidad Tecnológica de Bolívar.

9 Los contenidos temáticos, metodologías de enseñanza y referencias bibliográficas de los programas de los cursos no están completos para todos los programas de Economía de la muestra. El análisis de esto corresponde a otro proceso del proyecto de investigación. 
La estructura de los programas de Economía analizados sugiere cierta convergencia en el número total de cursos o asignaturas requeridas para obtener el título de economista pero con la presencia de algunas diferencias en el ámbito regional. De esta forma se tiene que la media es 48,67 asignaturas y la mediana es 47. Esto induce a pensar que hubo una reducción del número de asignaturas comparado con el resultado de González (1999) de 57 asignaturas en promedio con una mediana de $59^{10}$.

No existen diferencias significativas entre el número de cursos de los programas de Economía de universidades privadas $(48,65)$ y las universidades públicas $(48,75)$, pero es levemente mayor el número de cursos de los programas no acreditados por el CNA $(49,61)$ y los acreditados de alta calidad $(48,09)$.

Ahora bien, donde se observan diferencias significativas en el número de asignaturas es entre regiones del país. En la tabla 2 se puede observar que los programas de Economía de las regiones Central y Eje Cafetero tienen un menor número promedio de asignaturas comparado con las otras dos regiones. Lo anterior implica y reafirma la existencia de diferencias regionales entre los programas de Economía con relación a la estructura curricular.

Tabla 2. Número de asignaturas promedio de los programas de Economía por categoría y región

\begin{tabular}{|l|c|c|r|r|c|}
\multicolumn{1}{|c|}{ Región } & Promedio & Acreditadas & $\begin{array}{r}\text { No } \\
\text { acreditadas }\end{array}$ & Pública & Privadas \\
\hline Costa Atlántica & 51,16 & 50,75 & 52 & 53 & 50,25 \\
Central & 47,76 & 47,77 & 47,75 & 46 & 47,91 \\
Eje Cafetero & 45,50 & 44 & 47 & 46 & 45,25 \\
Suroccidental & 50,44 & 49 & 52,25 & 48,66 & 51,33 \\
\hline
\end{tabular}

Fuente: Base de datos del proyecto Estado de la Enseñanza de la Economía en Colombia, cálculos propios.

También se perciben particularidades intrarregionales con respecto al número de asignaturas de los programas de Economía. Los programas de Economía acreditados de alta calidad poseen significativamente menos asignaturas en promedio que los no acreditados en las regiones establecidas, a excepción de la región Central donde no hay diferencias en el número de asignaturas. Mientras tanto no se encuentra un patrón de diferencias preciso entre el número de asignaturas de los programas de las universidades públicas con respecto a los programas de las universidades privadas en las distintas regiones.

Esto implica que los programas de mayor calidad relativa y avalada por el CNA presentan sistemáticamente menos asignaturas que los no certificados, a pesar de tender o converger a un mismo número de semestres de estudio.

\subsection{Principales rasgos de las áreas temáticas}

\subsection{1 Área de microeconomía}

El área microeconómica comprende las asignaturas de microeconomía básica, intermedia y avanzada presentes en el currículo. Aquí se incluyen asignaturas 
como introducción a la economía, microeconomía I, microeconomía II, teoría del bienestar, equilibrio general, teoría de la firma, estructura de mercados y teoría de juegos.

En Colombia, la constante es que la secuencia de cursos básicos en el área de microeconomía está constituida por al menos un curso de fundamentos o principios de economía, y un ciclo de 3, 4 o 5 cursos de microeconomía. los cursos de microeconomía generalmente incluyen un primer nivel en algunos casos denominado introducción a la microeconomía en el que se aprenden los conceptos básicos del análisis microeconómico, tanto por el lado de la oferta como por el lado de la demanda, un curso en el que se profundizan los conocimientos adquiridos en el primer nivel y se introduce a los estudiantes en el análisis de equilibrio general, y uno o dos cursos en los que estudia el funcionamiento de diferentes estructuras de mercado con competencia imperfecta de la mano del estudio de la teoría de juegos para entender la incidencia de diversas estructuras de información en las decisiones de agentes económicos que interactúan entre sí.

Los programas de Economía analizados presentan un promedio de 4,15 asignaturas del área de microeconomía con una desviación estándar de 1,02 asignaturas y una mediana de cuatro asignaturas. Existe una pequeña diferencia entre los programas ofrecidos por universidades públicas (4,37 asignaturas) y los programas ofrecidos por las universidades privadas $(4,07)$ que coincide con la estrecha ventaja en participación del área de microeconomía en el currículo por parte de las universidades públicas encontrado por Lora y Ñopo (2009). En el caso de Colombia, estos autores encontraron -para una muestra de 13 instituciones públicas y 40 instituciones privadas- que en promedio $32 \%$ de las asignaturas de los programas de Economía de las universidades públicas corresponden a materias en esta área; entre tanto 30\% de las asignaturas de los programas de Economía de las universidades privadas corresponde a materias en la misma área.

El número promedio de asignaturas del área microeconómica por regiones no presenta mayores diferencias y se observa que fluctúa alrededor de las cuatro asignaturas" ${ }^{11}$ Sin embargo, se tiene una diferencia entre los programas acreditados (4,38 asignaturas) con respecto a los programas no acreditados (3,76 asignaturas). Esa diferencia del número de asignaturas del área microeconómica de los programas no acreditados se hace mayor cuando se compara con el número para los nueve programas de mejores resultados promedio en los ECAES - en adelante, el ranking de los programas top- $(4,77 \text { asignaturas })^{12}$.

Por lo tanto, los programas con altos niveles de calidad -según los resultados de los exámenes ECAES- tienden a tener una formación con mayor cantidad de asignaturas de microeconomía. Esto da indicios de la importancia de una buena fundamentación en esta área en la actualidad: una buena formación en un ciclo básico de la carrera de Economía exige el dominio de los conceptos e

11 El cálculo del índice de entropía muestra un resultado para las asignaturas del área de microeconomía de 0,012, para el área de macroeconomía es 0,029, para el área cuantitativa es 0,005 y para el área de historia y pensamiento es 0,092. Esto indica que existe bastante similitud en el número de asignaturas y su participación dentro de la estructura curricular.

12 Se realizó un ranking de los nueve programas con mejores resultados del examen de estado entre 2004 y 2010. Se escogieron nueve programas dado que el programa de la Escuela Colombiana de Ingeniería no pertenece a la muestra seleccionada. 
instrumentos analíticos propios de la microeconomía a un nivel básico, los cuales también son indispensables para alcanzar un buen manejo de los cursos de las demás áreas, en especial del área de macroeconomía -incluyendo el estudio las políticas económicas- e incluso del área de pensamiento económico. Internacionalmente, la importancia del área de microeconomía se expresa en la existencia de una mayor homogeneidad y estabilidad en los contenidos curriculares y en el material bibliográfico utilizado con respecto al área de macroeconomía (Gärtner 2001; Lora y Ñopo 2009).

\subsection{2 Área de macroeconomía}

El área de macroeconomía exhibe resultados similares al área microeconómica. Para la definición de esta área se tuvieron en cuenta las asignaturas de fundamentos de macroeconomía, macroeconomía I, II y III (en algunos casos), macroeconomía internacional, política fiscal y política monetaria. El promedio de asignaturas es 4 y la mediana 4 , que coincide con el promedio de los programas top. Existen leves diferencias entre los programas de las universidades privadas $(4,15)$ con respecto a las universidades públicas $(3,63)$, y entre los programas acreditados $(3,9)$ y los no acreditados $(4,23)$. Los resultados regionales muestran fluctuación alrededor de las 4 asignaturas, con resultados algo diferentes para la región Central y la Costa Atlántica, tal como se puede apreciar en la tabla 3.

\subsection{3 Área cuantitativa}

Para el área cuantitativa existe convergencia en el número de asignaturas, alrededor de ocho, y no existen significativas diferencias entre las distintas tipologías aquí presentadas. La única consideración se refiere a que el número de asignaturas es más alto en comparación con las demás áreas de análisis, tal como se puede observar en la tabla 3. La definición de esta área tiene en cuenta las asignaturas de matemáticas, estadística, econometría, métodos cuantitativos y economía matemática.

Esto confirma la importancia que la mayoría de los programas de Economía en Colombia le dan hoy en día a la formación cuantitativa de sus estudiantes. Esto es consistente con las tendencias globales en la enseñanza de la economía y es resultado de los enormes desarrollos de los métodos cuantitativos - tanto estadísticos como matemáticos y también computacionales- en la disciplina. La aplicación de estos métodos, que en realidad ha sido tradicional en economía desde mediados del siglo pasado, ha ganado importancia recientemente debido al incremento vertiginoso de la información disponible en grandes bases de datos, en una nueva era en la que el avance de las comunicaciones y la disponibilidad de datos a gran escala (big data) han transformado las dimensiones en que puede desarrollarse el trabajo empírico en todas las ramas de la ciencia, sin excluir a las ciencias sociales.

Tanto las vertientes ortodoxas que pertenecen al mainstream como las distintas escuelas heterodoxas de economía han venido utilizando durante las últimas décadas una cantidad creciente de métodos cuantitativos cada vez más diversos, potentes y sofisticados. Aunque esto es más notorio en la investigación 
y cursos en los programas doctorales y de maestría, los programas de pregrado han comenzado a verse influidos durante las últimas décadas por la necesidad de implementar nuevas y mejores rutinas de carácter cuantitativo.

\subsection{4 Áreas de pensamiento económico e historia}

El área de pensamiento económico registra significativas diferencias a favor de los programas de Economía ofrecidos por las universidades públicas y los programas acreditados por el CNA. De hecho, los programas de las universidades públicas muestran un promedio de 3,75 asignaturas frente a 1,85 de los programas de las universidades privadas para la muestra de análisis de este trabajo. Incluso el promedio de asignaturas de estos últimos programas está por debajo del promedio general $(2,09)$ y del promedio de los programas del ranking de los programas top. Asimismo, los programas acreditados revelan una diferencia pequeña pero significativa en el número promedio de asignaturas de esta área con respecto a los programas no acreditados (tabla 3).

Tabla 3. Número de asignaturas y participación promedio de las áreas temáticas de los programas de Economía por categoría y región

\begin{tabular}{|l|r|r|r|r|r|r|r|r|}
\hline \multirow{2}{*}{ Area } & \multicolumn{3}{|c}{ Típo } & \multicolumn{1}{c|}{ Acreditación } & \multicolumn{4}{c|}{ Región } \\
\cline { 2 - 11 } & Privada & Pública & \multicolumn{1}{c|}{ Si } & No & Central & Eje C. & Costa & Suroc. \\
\hline \multirow{2}{*}{ Microeconomía } & 4,07 & 4,37 & 4,38 & 3,76 & 4,07 & 4,50 & 4,16 & 4 \\
& $8,50 \%$ & $9,10 \%$ & $9,20 \%$ & $7,70 \%$ & $8,70 \%$ & $10 \%$ & $8,20 \%$ & $8,10 \%$ \\
\hline Macroeconomía & 4,15 & 3,63 & 3,90 & 4,23 & 3,69 & 4 & 4,83 & 4 \\
& $8,60 \%$ & $7,50 \%$ & $8,30 \%$ & $8,50 \%$ & $7,80 \%$ & $8,80 \%$ & $9,60 \%$ & $8,10 \%$ \\
\hline Cuantitativa & 8,15 & 7,88 & 8,10 & 8 & 8 & 8,50 & 7,80 & 8,10 \\
& $16,80 \%$ & $16,30 \%$ & $17 \%$ & $16,20 \%$ & $16,70 \%$ & $18,70 \%$ & $15,50 \%$ & $16,10 \%$ \\
\hline Pensamiento & 1,58 & 3,75 & 2,24 & 1,85 & 1,69 & 2,16 & 2,16 & 2,55 \\
& $3,20 \%$ & $7,70 \%$ & $4,60 \%$ & $3,70 \%$ & $3,40 \%$ & $4,80 \%$ & $4,20 \%$ & $5,20 \%$ \\
\hline Historia & 1,35 & 2 & 1,71 & 1,15 & 1,53 & 1,16 & 1,50 & 1,16 \\
& $2,80 \%$ & $4,10 \%$ & $3,60 \%$ & $2,30 \%$ & $3,30 \%$ & $2,60 \%$ & $2,90 \%$ & $3,30 \%$ \\
\hline
\end{tabular}

Fuente: Base de datos del proyecto Estado de la Enseñanza de la Economía en Colombia, cálculos propios.

Las asignaturas tenidas en cuenta fueron para la especificación de esta área correspondieron a: pensamiento económico, historia del pensamiento económico, historia económica general, historia económica de Colombia y similares o relacionadas.

Otro hecho significativo es que existen diferencias regionales con relación al número promedio de asignaturas de la estructura curricular de los programas de Economía dedicado al área de pensamiento económico. De esta manera, la región Central tiene un número promedio de 1,69, mientras la Suroccidental exhibe un promedio de 2,56 asignaturas. Cabe resaltar el impacto que tienen las universidades del Cauca y Nariño, dado que son los programas con más alto número de asignaturas de esta área.

Otra área de análisis es historia económica. La sistematización de la información recolectada muestra que el promedio de asignaturas para toda la muestra 
es 1,5 asignaturas y una mediana de dos asignaturas. Lo más común es que los programas contengan dos asignaturas de historia económica: una sobre historia económica general y la otra sobre historia económica de Colombia. Son previsibles las diferencias entre los programas acreditados $(1,71)$ y no acreditados $(1,15)$, y entre los programas ofrecidos por universidades públicas (2) y las universidades privadas $(1,35)$, teniendo en cuenta lo observado en el área de pensamiento económico.

Los dos resultados anteriores indican que los programas de Economía de las universidades acreditadas y los de las universidades públicas tienden a ofrecer una formación más integral, en la que tiene cabida la formación en historia económica e historia del pensamiento económico.

\subsubsection{Otras áreas curriculares}

Para completar el análisis de este trabajo se definieron y manejaron tres áreas curriculares adicionales (en adelante otras áreas). La primera corresponde a los saberes específicos en economía que incluye las asignaturas obligatorias del ciclo de profundización y profesionalización propios en teoría y política económica y en economía aplicada. Son asignaturas que afianzan los conocimientos y competencias de los estudiantes en áreas de manejo y experticia propias de los economistas, que se encuentran en la estructura curricular y son obligatorias cursar para optar al título de economista.

$\mathrm{Al}$ respecto, los resultados muestran que los programas de las universidades públicas ofrecen más asignaturas en promedio $(12,1)$ que los programas de las universidades privadas $(9,27)$. Asimismo, existen marcadas diferencias regionales dado que, por un lado, la región Suroccidental $(7,56)$ y Eje Cafetero $(7,67)$ están por debajo del promedio total $(9,94)$ y la mediana (9) y, por otro lado, la región Central $(10,9)$ y Costa Atlántica $(13,7)$ están por encima de dichos valores (tabla 4).

La segunda área definida es "otros saberes" que comprende las asignaturas de la estructura curricular complementarias al saber y la aplicación de la economía y que contribuyen a la formación integral del economista. Formación integral entendida como un proceso que busca el estímulo de la creatividad y la

Tabla 4. Número de asignaturas y participación promedio de las áreas "propuestas" de los programas de Economía por categoría y región

\begin{tabular}{|c|c|c|c|c|c|c|c|c|}
\hline \multirow{2}{*}{ Área } & \multicolumn{2}{|c|}{ Tipo } & \multicolumn{2}{|c|}{ Acreditación } & \multicolumn{4}{|c|}{ Región } \\
\hline & Privada & Pública & Si & No & Central & Eje C. & Costa & Suroc. \\
\hline $\begin{array}{l}\text { Saberes } \\
\text { específicos en } \\
\text { economía }\end{array}$ & $\begin{array}{r}9,20 \\
18,90 \%\end{array}$ & $\begin{array}{r}12,10 \\
24 \%\end{array}$ & $\begin{array}{l}9,90 \\
20 \%\end{array}$ & $\begin{array}{r}10 \\
19,70 \%\end{array}$ & $\begin{array}{r}10,90 \\
22,60 \%\end{array}$ & $\begin{array}{r}7,60 \\
16,50 \%\end{array}$ & $\begin{array}{r}13,60 \\
25,90 \%\end{array}$ & $\begin{array}{r}7,50 \\
14,90 \%\end{array}$ \\
\hline Otros saberes & $\begin{array}{r}12,60 \\
25,40 \%\end{array}$ & $\begin{array}{r}8,80 \\
18,40 \%\end{array}$ & $\begin{array}{r}10,10 \\
20 \%\end{array}$ & $\begin{array}{r}14,40 \\
28,60 \%\end{array}$ & $\begin{array}{r}10,50 \\
21,60 \%\end{array}$ & $\begin{array}{l}8,60 \\
19 \%\end{array}$ & $\begin{array}{r}12 \\
23,70 \%\end{array}$ & $\begin{array}{r}15,40 \\
30,10 \%\end{array}$ \\
\hline Flexible & $\begin{array}{r}5,80 \\
11,10 \% \\
\end{array}$ & $\begin{array}{r}5,30 \\
11,60 \% \\
\end{array}$ & $\begin{array}{r}6,40 \\
13,20 \% \\
\end{array}$ & $\begin{array}{r}4,30 \\
7,40 \% \\
\end{array}$ & $\begin{array}{r}8,30 \\
18,20 \% \\
\end{array}$ & $\begin{array}{r}4,80 \\
10,70 \% \\
\end{array}$ & $\begin{array}{r}2,20 \\
3 \% \\
\end{array}$ & $\begin{array}{r}4,20 \\
7,50 \% \\
\end{array}$ \\
\hline
\end{tabular}

Fuente: Base de datos del proyecto Estado de la Enseñanza de la Economía en Colombia, cálculos propios. 
responsabilidad, el conocimiento interdisciplinario y el desarrollo de capacidades de análisis complejo.

Después de sistematizar la información de la muestra al respecto se puede afirmar que existen diferencias significativas entre las distintas tipologías de los programas de Economía con respecto al área de "otros saberes". De esta forma, mientras los programas acreditados tienen en promedio 10,1 asignaturas en esta área, los programas no acreditados presentan un promedio de 14,46 asignaturas. Ambos tipos de programas están por encima de la media de los programas del ranking 9, que exhiben 7,77 asignaturas de este tipo en promedio. También existen marcadas diferencias entre los programas de universidades privadas $(12,65)$ frente a los de universidades públicas $(8,87)$ y en el ámbito regional como se observa en la tabla 4 .

La última área curricular definida se ha denominado "área flexible". Acá se consideran las asignaturas de tipo electivas o seminarios profesionales de la estructura curricular de los programas de Economía que se cursan de manera obligatoria para completar los requisitos para obtener el título de economista pero que son de libre escogencia entre una oferta o catálogo por parte de los estudiantes. Las electivas o seminarios buscan profundizar los conocimientos y competencias en economía y se dirigen, en su mayoría, a formular y plantear preguntas y problemas en economía.

Este componente flexible presenta una media de 5,7 asignaturas y una mediana de 4; valores inferiores al promedio del ranking 9 que es 8,77 asignaturas. Acá también se observan diferencias significativas entre los programas acreditados $(6,45)$ y no acreditados $(4,3)$ y en el ámbito regional. Es importante observar la gran diferencia entre el ofrecimiento promedio de los programas de la región Central $(8,33)$ frente a las demás regiones especialmente la Costa Atlántica $(2,25)$.

Esto indica que los programas de la región Central, especialmente Bogotá, ofrecen mayores oportunidades y una más amplia oferta relativa para afianzar los conocimientos básicos y profesionales en economía o áreas afines, tal vez por su nivel de desarrollo económico y un mayor número de profesionales en economía, investigadores, grupos y centros de investigación.

\section{Comentarios finales}

Durante los últimos 15 años se ha presentado un flujo importante de creación de nuevos programas de Economía y desaparición de otros. Hoy en día, el número se encuentra alrededor de 75 programas focalizados principalmente en las tres principales ciudades del país (52\%) y especialmente en Bogotá (33\%).

De acuerdo con los resultados de los análisis de la estructura curricular de los programas de Economía escogidos para este trabajo se encuentra que existe cierta convergencia a una duración de nueve semestres y un número de 48 asignaturas, no obstante unas pequeñas diferencias regionales. De igual manera se encuentra convergencia en el número de asignaturas de las áreas de microeconomía, macroeconomía y cuantitativo entre los currículos de la muestra analizada.

Estos resultados demuestran convergencia y homogenización de currículos en cuanto al número de asignaturas y su participación relativa de las áreas microeconómica, macroeconómica y cuantitativa. Además esos resultados coinciden en sus tendencias con los encontrados por Lora y Ñopo (2009). 
Esta creciente homogenización de currículos puede sugerir la existencia de una estrategia de líder-seguidor dentro de los programas de Economía. Los programas tienden a imitar la estandarización de unos programas líderes para ofrecer currículos "modernos", competentes, prestigiosos o de renombre, flexibles e integrales en algunos casos sin tener en cuenta sus propias especificidades o sellos distintivos o diferenciadores. No obstante, lo logran en parte debido a sus propias limitaciones y restricciones institucionales, de requerimientos de la demanda o de escasez relativa de profesionales competentes en ciertas áreas de la economía, como la historia y el pensamiento económico en algunas regiones del país.

Durante la década pasada no era tan marcada la tendencia hacia la homogeneización, pero como advertía Cataño (2004) ya en ese entonces saltaba a la vista la existencia de "una vanguardia de facultades de economía que sirven de referencia a las otras" (2004, 139). Estas facultades de prestigio o vanguardia sirven a las demás como un punto de referencia u óptimo local para ajustar sus programas académicos.

Además la "presión" académica generada a partir de los procesos de acreditación de alta calidad ha conllevado a que los programas de Economía tiendan a implementar esta estrategia como una forma de señalizar la calidad de los programas ofrecidos frente a los clientes potenciales (los estudiantes) y frente a las instituciones que regulan el sistema de calidad, en este caso el MEN, Conaces, el Consejo Nacional de Acreditación (CNA), la Asociación Colombiana de Facultades y Departamentos de Economía (Afadeco) y Colciencias.

Todo este marco institucional creado por los órganos y procesos de aseguramiento de la calidad configura un sistema de incentivos con premios y castigos claramente definidos, donde alejarse de los estándares puede ser costoso para los programas académicos. La razón de ser de este marco institucional es la información asimétrica connatural al ofrecimiento de servicios educativos en los mercados de educación. ¿Pero qué tan conveniente ha sido todo este proceso? Esta es una pregunta sobre la que cabría pensar más adelante y de forma amplia en otros trabajos.

Por otra parte, se observan diferencias y divergencias significativas en las áreas de pensamiento e historia económica, al igual que en la capacidad de ofrecimiento de flexibilidad y profundización en economía, tal como se mostró anteriormente.

Sería importante en el futuro analizar más detenidamente el efecto de los procesos de acreditación de alta calidad, porque al parecer estos tienden a homogenizar los currículos, aunque les están bridando importancia a las áreas de pensamiento e historia económica. Esto último es interesante y va en la dirección de los "modelos ideales" de enseñanza de la economía a los que hicimos referencia en la segunda sección de este artículo.

Sin embargo, consecuencias negativas del modelo actual como la falta de identidad de algunos programas y los niveles insuficientes de integralidad e interdisciplinaridad en la mayoría de los programas también deberían ser estudiadas a fondo en próximos trabajos. Por último, también es necesario estudiar más detalladamente las temáticas manejadas para dar cuenta de la pluralidad en la enseñanza desde la perspectiva de teorías, modelos y metodologías alternativas de estudio y referencia. 


\section{Referencias bibliográficas}

Ahumada, Ivico y Fernando Butler. «La enseñanza de la economía en México». BID Research Departament Working papers, nº 672, 2009: 1-68.

Bejarano, Jesús. "Los límites del conocimiento económico y sus implicaciones pedagógicas». Cuadernos de Economía, Vol. 6, nº 6, 1984: 35-59.

Cataño, José. «La ciencia económica actual y la enseñanza de la Economía: el debate francés». Lecturas de Economía, nº 60, 2004: 121-139.

Cuenca, María. «La enseñanza en Economía: una reflexión desde la perspectiva del contenido». Semestre económico, Vol. 13, n 26, 2010: 135-147.

Currie, Lauchlin. La enseñanza de la Economía en Colombia. Bogotá: Tercer Mundo Editores, 1965.

Currie, Lauchlin. «La enseñanza de la economía II: extractos de informes sobre algunas facultades». Cuadernos de Economía, Vol. XIII, n 18-19, 1993: 345-354.

D'Autume, Antoine. La querelle sur l'enseignement de l'économie, Sortir par le haut. Real-World Economics Review, 2000. www.paecon.net (último acceso: 17 de mayo de 2015).

De Camilloni, Alicia. «Estándares, evaluación y currículo». Archivos de Ciencias de la Educación, $\mathrm{n}^{\circ}$ 3, 2009: 55-68.

Espinoza, Lourdes, Carlos Machicado y Katia Makhlouf. «La enseñanza de Economía en Bolivia y Chile». BID Research Departament Working papers, $\mathrm{n}^{\circ}$ 673, 2009: 1-142.

Fourcade, Marion, Etienne Ollion y Yann Algan. «The Superiority of Economists». The Journal of Economic Perspectives, Vol. 29, $\mathrm{n}^{\circ}$ 1, 2015: 89-113.

Gärtner, Manfred. «Teaching Economics to Undergraduates in Europe: Volume, Structure, and Contents». The Journal of Economic Education, Vol. 32, $\mathrm{n}^{\mathrm{o}} 3$, 2001: 219-230.

González, Jorge. «La fetichización del currículo y la absolutización del libro de texto». En Hacia dónde va la ciencia económica en Colombia. Siete ensayos exploratorios, de Jesús Bejarano, 53-88. Bogotá: Tercer Mundo Editores, 1999.

Hodara, Isidoro. La enseñanza de la economía en América Latina. Montevideo: Universidad ORT Uruguay, 2010. www.ort.edu.uy/facs/pdf/hodaraort1010.pdf (último acceso: 17 de febrero de 2015).

Kalmanovitz, Salomón. «El debate debe continuar. Bejarano y la enseñanza de la Economía». Cuadernos de Economía, Vol. 18, nº 31, 1999: 173-198.

Lora, Eduardo y Hugo Ñopo. «La formación de los economistas en América Latina». Revista de análisis económico, Vol. 24, $\mathrm{n}^{\circ}$ 2, 2009: 65-93.

Raffo, Leonardo y Javier Castro. «Los problemas fundamentales de la economía walrasiana y su influencia en la enseñanza de la Economía». Documentos del Trabajo del CIDSE, $\mathrm{n}^{\circ}$ 135, 2011: 1-30.

Rozenwurcel, Guillermo, Gabriel Bezchinsky y Marisol Rodríguez. «La enseñanza de Economía en Argentina». BID Research Departament Working papers, $\mathrm{n}^{\circ}$ 671, 2009: 1-79.

Sábato, Ernesto. Apologías y rechazos. Barcelona: Seix Barral, 1979.

Salazar, Boris. Currículos, pedagogía y paradigmas: ¿es posible saltar a las fronteras para aprender más? Cali: Universidad del Valle (mimeo), 2010. 
Sarmiento, Jaime y Carolina Silva. «La formación del economista en Colombia». Investigación y Reflexión, $\mathrm{n}^{\circ}$ 1, 2014: 231-262.

Villanueva, Ernesto. «Aseguramiento de calidad y convergencia de la educación superior en América Latina». En Movilidad, aseguramiento de la calidad y reconocimiento mutuo de títulos de 10 educación superior en América Latina, coordinado por Sylvie Diduo, 47-63. México D.F.: Unesco- México, 2008.

Weintraub, E. Roy. General Equilibrium Analysis: Studies in Appraisal. Cambridge: Cambridge University Press, 1985.

\section{Documentos oficiales}

Presidencia de la República de Colombia. Decreto 2230 de 2003. Bogotá D.C.: Presidencia de la República de Colombia, 2003. 